\title{
Study on a collecting structure for impact energy and its dynamic characteristics
}

\author{
Kai Zhang *, Yongyong Zhu and Qizheng Zhou \\ Dept. of Weaponry Engineering, Naval Univ. of Engineering, Wuhan, China
}

\begin{abstract}
The impact energy harvesting technology is studied in this paper, a piezoelectric harvesting structure with additional resonant structure including spring mass components is proposed, and the system dynamics model of the structure is studied. The influence of the structural parameters of spring mass block on the system output voltage is studied by using the model. The research results have certain application value for impact energy harvesting.
\end{abstract}

\section{Introduction}

The piezoelectric harvesting for impact energy is a hot research direction in the field of energy collection technology[1,2,3]. Because the impact character of shorttime and instantaneous, which is different from the collection of continuous vibration energy, the energy conversion and storage time for the impact energy harvesting device is limited by the impact pulse width[4]. The intermittent pulse nature of impact response means that it contains rich frequencies. In contrast, The vibration is continuous, and the continuous supply of vibration energy makes the transducer and collection circuit have enough time for energy conversion, collection and storage. Therefore, collecting impact energy is more difficult than collecting vibration energy.

In the collection of single impact energy, the adverse effects of impact time and excitation frequency should be considered. Apparently, the process of energy conversion can be maintained by prolonging the action time of excitation force and changing the natural frequency of the whole vibration system, so as to optimize the system parameters to improve the energy collection efficiency. Therefore, a buffer device is designed in this paper, which can store the transient and impact energy in the form of displacement energy, and then release it slowly. The working principle of this structure is similar to that of the vibration absorber. It is mainly composed of hard springs and mass blocks. During vibration, it can be regarded as an additional resonator to change the resonance frequency of the energy collector.

Under the impact action, the structure converts the impact energy into the kinetic energy of the mass and the elastic potential energy of the spring, and uses the reciprocating motion of the spring to transform the single pulse impact load into multi pulse excitation. In addition, by changing the design parameters of the spring mass block, the resonator frequency is adjusted so that the resonance frequency of the resonator is close to that of the transducer, so as to achieve the purpose of multi-modal energy collection. Compared with the simple piezoelectric structure, the additional resonant structure also brings great flexibility to the design of energy collector.

\section{Structural design}

In order to realize the collection of impact energy, by fixing the spring-mass block assembly on the end cap of the piezoelectric transducer, in this way, the mass will displace when impacted, the impact energy will be transformed into the kinetic energy of the mass and the potential energy of the spring. In the reciprocating motion of the mass, the piezoelectric transducer intermittently outputs electric energy. The piezoelectric transducer adopts cymbal structure to improve the electromechanical conversion efficiency[5,6]. The structural diagram is shown in the figure1. In addition, changing the design parameters of spring-mass block can adjust the resonance state of energy harvester, which can realize multi-modal energy harvesting.

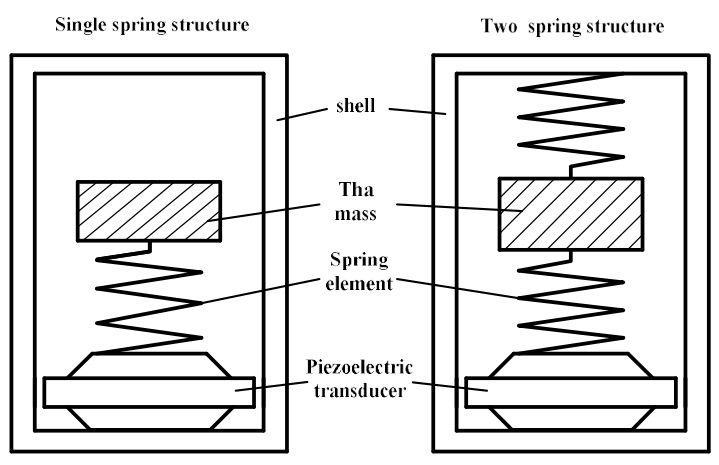

Figure 1. Design of impact energy harvester

\footnotetext{
$\overline{\text { * Corresponding author: zys } 88488 @ 163 . c o m}$
} 


\section{Dynamic model}

The dynamic model of the impact energy harvester is shown in Figure 2. $m_{1} 、 c_{1} 、 k_{1}$ are the mass of mass block, spring stiffness and damping in the additional resonator respectively, $m_{2} 、 c_{2} 、 k_{2}$ are the equivalent mass, equivalent damping and piezoelectric structure stiffness of the piezoelectric transducer respectively, $x_{1}(t), x_{2}(t)$ are the displacement of $m_{1}$ and $m_{2}$. Considering that the load is pure resistance R, during the working process, External excitation to the piezoelectric transducer under the action of the impact force $F(t)$, and the generated voltage is $V$. The piezoelectric plate of the piezoelectric transducer is axially polarized. Assuming that the axial stress is $T_{3}$, the piezoelectric equation is adopted

$$
\begin{gathered}
S_{3}=s_{33}^{E} T_{3}+d_{33} E_{3} \\
D_{3}=d_{33} T_{3}+\varepsilon_{33}^{T} E_{3}
\end{gathered}
$$

Where, $S_{3}$ and $T_{3}$ are axial strain and axial stress respectively, $E_{3}$ and $D_{3}$ are axial electric field strength and electric displacement, $\varepsilon_{33}^{T}$ is the dielectric constant in free state, $S_{33}^{E}$ is the elastic compliance constant under constant electric field.

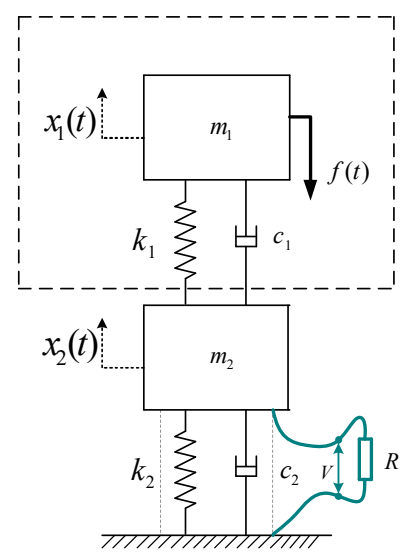

Figure 2. Model of piezoelectric impact energy harvester

Combined the equation (1) and equation (2), the electric displacement of piezoelectric vibrator is

$$
D_{3}=\frac{q}{A}=d_{33} c_{33}^{E} S_{3}+\frac{\left(1-k_{33}^{2}\right) \varepsilon_{33}^{T}}{h_{p}} v
$$

Further, the charge expression at both ends of the piezoelectric wafer is

$$
q=A d_{33} c_{33}^{E} S_{3}+C_{p} v
$$

Where, $A$ is the area of the piezoelectric disc, $V$ is the voltage at both ends of the electrode, $c_{33}^{E}=1 / s_{33}^{E}$ is the Young's modulus, $C_{p}=\left(1-k_{33}^{2}\right) c_{p}$ is the clamping capacitance, $\quad c_{p}=A \varepsilon_{33}^{T} / h_{p} \quad$ is the non-clamping capacitance and $k_{33}$ is the electromechanical coupling coefficient, $h_{p}$ is the thickness of the piezoelectric ceramic sheet.
According to the dynamic analysis, the differential equation of motion of the system can be expressed as

$$
\begin{aligned}
& {\left[\begin{array}{ccc}
m_{1} & 0 & 0 \\
0 & m_{2} & 0 \\
0 & 0 & 0
\end{array}\right]\left[\begin{array}{c}
\ddot{x}_{1}(t) \\
\ddot{x}_{2}(t) \\
\ddot{v}(t)
\end{array}\right]+\left[\begin{array}{ccc}
c_{1} & -c_{1} & 0 \\
-c_{1} & c_{1}+c_{2} & 0 \\
0 & d_{33} k_{2} & C_{p}\left(1-k_{33}^{2}\right)
\end{array}\right]\left[\begin{array}{c}
\dot{x}_{1}(t) \\
\dot{x}_{2}(t) \\
\dot{v}(t)
\end{array}\right]} \\
& +\left[\begin{array}{ccc}
k_{1} & -k_{1} & 0 \\
-k_{1} & k_{1}+k_{2} & d_{33} k_{2} \\
1 / R
\end{array}\right]\left[\begin{array}{c}
x_{1}(t) \\
x_{2}(t) \\
v(t)
\end{array}\right]=\left[\begin{array}{c}
f(t) \\
0 \\
0
\end{array}\right]
\end{aligned}
$$

Assuming that the sinusoidal half wave pulse acts on the mass $m_{1}$, the expression of the pulse force is

$$
f(t)=\left\{\begin{array}{lc}
f_{0} \sin \omega_{0} t & 0 \leq t \leq t_{1} \\
0 & t>t_{1}
\end{array}\right.
$$

Where $f_{0}$ is the amplitude of the force and $\omega_{0}$ is the angular frequency of the exciting force. Laplace transform equation (3) to obtain

$$
\begin{gathered}
\left(s^{2}+2 \zeta_{1} \omega_{1} s+\omega_{1}^{2}\right) X_{1}-\left(2 \zeta_{1} \omega_{1} s+\omega_{1}^{2}\right) X_{2}=L\left(a \sin \left(\omega_{0} t\right)\right) \\
X_{2}=-\frac{\left(C_{p} R s+1\right)}{R k_{2} d_{33} s} V \\
{\left[s^{2}+2 \zeta_{2} \omega_{2} s+\omega_{2}^{2}+\mu\left(2 \zeta_{1} \omega_{1} s+\omega_{1}^{2}\right)\right] X_{2}-\mu\left(2 \zeta_{1} \omega_{1} s+\omega_{1}^{2}\right) X_{1}+d_{33} \omega_{2}^{2} V=0}
\end{gathered}
$$

Then, combined the equation(4), (5) and (6), the follow expression can be obtained.

$V=-\left(2 \zeta_{1} \omega_{1} s+\omega_{1}^{2}\right) R a k_{2} d_{33} s^{2} /\left[\left(s^{2}+\omega_{0}^{2}\right)\left(a_{5} s^{5}+a_{4} s^{4}+a_{3} s^{3}+a_{2} s^{2}+a_{1} s+a_{0}\right)\right]$

Where,

$$
\begin{aligned}
& a_{5}=\mu C_{p} R \\
& a_{4}=\mu+C_{p} R\left(2 \mu \zeta_{2} \omega_{2}+2(\mu+1) \zeta_{1} \omega_{1}\right) \\
& =\mu R\left(C_{p}-k_{2} d_{33}^{2}\right) \omega_{2}^{2}+(\mu+1) C_{p} R \omega_{1}^{2}+4 \mu R C_{p} \zeta_{1} \zeta_{2} \omega_{1} \omega_{2} \\
& a_{2}=2 \mu R\left[\left(C_{p}-k_{2} d_{33}^{2}\right) \zeta_{1} \omega_{1} \omega_{2}^{2}+C_{p} \zeta_{2} \omega_{2} \omega_{1}^{2}\right]+\mu \omega_{2}^{2}+4 \mu R C_{p} \zeta_{1} \zeta_{2} \omega_{1} \omega_{2}+(\mu+1) \omega_{1}^{2} \\
& a_{1}=\left(C_{p}-k_{2} d_{33}^{2}\right) \mu R \omega_{1}^{2} \omega_{2}^{2}+2 \mu \zeta_{1} \omega_{1} \omega_{2}^{2}+2 \mu \zeta_{2} \omega_{2} \omega_{1}^{2} \\
& a_{0}=\mu \omega_{1}^{2} \omega_{2}^{2} \quad \omega_{2}^{2}=k_{2} / m_{2}, \\
& \mu=m_{2} / m_{1}, \quad \omega_{1}^{2}=k_{1} / m_{1} \quad, \quad \omega_{2} \\
& \zeta_{1}=c_{1} / 2 \sqrt{k_{1} m_{1}}, \zeta_{2}=c_{2} / 2 \sqrt{k_{2} m_{2}} .
\end{aligned}
$$

When $t>t_{1}, F(t)=0$. At that time, the system was affected by the initial displacement and system damping, and the system began to have damped free vibration. The system output voltage in the frequency domain can be calculated by equation (7). The influence of additional spring mass block components on the output voltage can be investigated by changing the spring stiffness, mass block mass, damping coefficient and other parameters.

\section{Numerical calculation and analysis}

According to the established dynamic model, the effects of the masses $m_{1}, k_{1}$ and $c_{1}$ in the additional resonator on the system output voltage are investigated. The main parameters in the simulation are selected as follows:

PZT-5A $\quad\left(d_{33}=374 \times 10^{-12} \mathrm{~m} / \mathrm{V}, s_{33}^{E}=18.8 \times 10^{-12} \mathrm{~m}^{2} / \mathrm{N}\right.$, 
$\left.\varepsilon_{33}^{T}=1700\right), a=3000 \mathrm{~g}, A=4.5 \times 10^{-4} \mathrm{~m}^{2}, t_{\mathrm{p}}=0.01 \mathrm{~m}, R=1 \mathrm{M}$

$\Omega$.

Firstly, the influence of spring stiffness on the output voltage is investigated. On the basis of not changing the structural parameters and material parameters of piezoelectric transducer, $m_{1}=0.02 \mathrm{~kg}, c_{2}=0.05, R=1 \mathrm{M} \Omega$ are selected, and $k_{1}$ are seted on $3500 \mathrm{~N} / \mathrm{m}, 5000 \mathrm{~N} / \mathrm{m}$, $10000 \mathrm{~N} / \mathrm{m}$ and $20000 \mathrm{~N} / \mathrm{m}$ respectively. The output voltage curve is obtained as shown in Figure.3. It can be seen from the figure that when the exciting force frequency $\omega_{0}=100 \mathrm{rad} / \mathrm{s}$, the first-order frequency does not change with the increase of spring stiffness, the second-order frequency gradually shifts to high frequency, and the second-order vibration peak gradually decreases. When $\omega_{0}=1000 \mathrm{rad} / \mathrm{s}$, the first-order frequency gradually shifts to high frequency, the second-order frequency does not change.

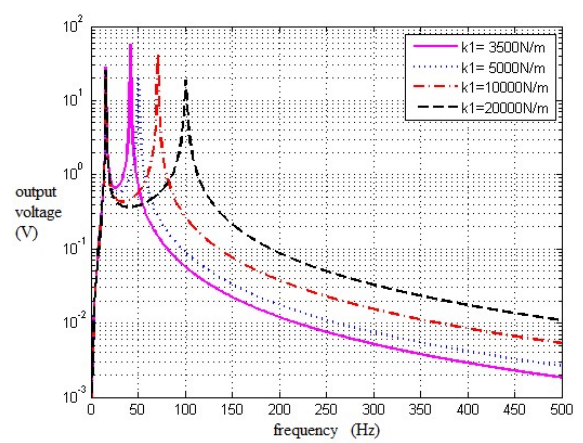

(a) $\omega_{0}=100 \mathrm{rad} / \mathrm{s}$

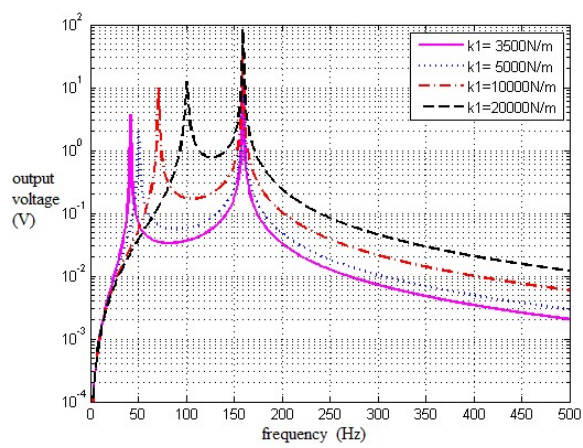

(b) $\omega_{0}=1000 \mathrm{rad} / \mathrm{s}$

Figure 3. Output voltage curve of different spring stiffness k1

The influence of mass on the system output voltage is investigated. Considering $k_{1}=10000 \mathrm{~N} / \mathrm{m}$, and $0.02 \mathrm{~kg}$, $0.05 \mathrm{~kg}, 0.08 \mathrm{~kg}$ and $0.15 \mathrm{~kg}$ are selected for $m_{1}$ respectively. The corresponding output voltage curve is shown in Figure.4. when $\omega_{0}=100 \mathrm{rad} / \mathrm{s}$, the first-order resonance, frequency is $20 \mathrm{~Hz}$, and the second-order common vibration frequency decreases with the increase of $m_{1}$. When $\omega_{0}=1000 \mathrm{rad} / \mathrm{s}$, the second-order resonance frequency was about $165 \mathrm{~Hz}$, and the first-order resonance frequency increased with the increase of $m_{1}$.

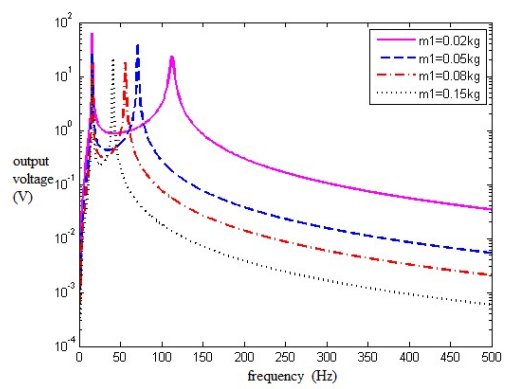

(a) $\omega_{0}=100 \mathrm{rad} / \mathrm{s}$

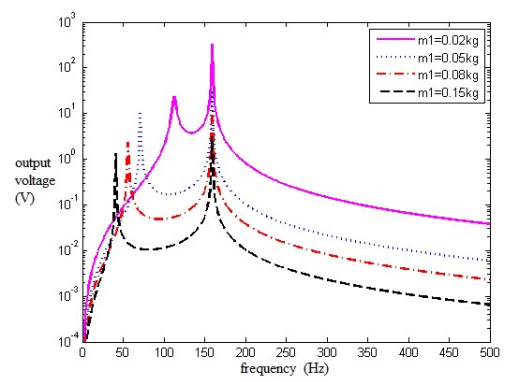

(b) $\omega_{0}=1000 \mathrm{rad} / \mathrm{s}$

Figure 4. Output voltage curve of different mass $m_{1}$

Similarly, considering $k_{1}=10000 \mathrm{~N} / \mathrm{m}$, and the damping coefficient $c_{1}$ are $0.1,0.3,0.5$ and 0.8 respectively. As shown in Figure.5, with the increase of damping coefficient, the second-order resonance peak decreases gradually. When $\omega_{0}=100 \mathrm{rad} / \mathrm{s}$, the second-order peak voltage of the system corresponding to $c_{1}=0.1$ and $c_{1}=$ 0.5 is $50.73 \mathrm{~V}$ and $9.79 \mathrm{~V}$ respectively. At this time, the second-order resonance frequency is $71 \mathrm{~Hz}$. When $\omega_{0}=1000 \mathrm{rad} / \mathrm{s}, c_{1}$ only affected the first-order resonance peak.

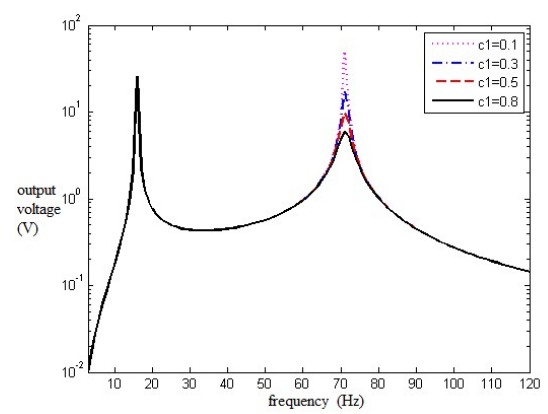

(a) $\omega_{0}=100 \mathrm{rad} / \mathrm{s}$

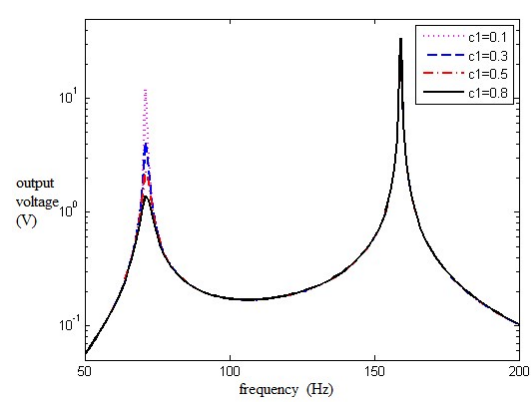

(b) $\omega_{0}=1000 \mathrm{rad} / \mathrm{s}$

Figure 5. Output voltage curve of different damper $c_{1}$ 


\section{Conclusion}

Based on the design of the impact energy harvester, the electromechanical coupling dynamic model of piezoelectric impact energy harvester with additional resonator is derived, the frequency domain expression of system output voltage is given, and the effects of the resonator structural parameters, excitation frequency and load resistance on the output piezoelectric performance of the system are discussed, The conclusions are as follows: the additional spring mass module changes the vibration characteristics of the piezoelectric energy capture system and makes the piezoelectric energy capture appear second-order resonance; Increasing the excitation frequency can shift the first-order resonant frequency to the second-order resonant frequency, and the first-order resonant peak also increases.

\section{References}

1. Ali Mohammed Abdal-Kadhim, Kok Swee Leong, Impact-driven energy harvesting:effect of stress on piezoelectric bender. J. Tel. Ele and Comp.Eng, 10(2018)87-92.

2. S. Ju, C.H. Ji, R.A. Lupton, Impact-based piezoelectric vibration energy harvester, Applied Energy. 214(2018) 139-151.

3. J. Caban, G. Litak. Impact-based piezoelectric vibration energy harvesting system excited from diesel engine suspension. Applied computer science, 16(2020)16-29.

4. E. Köhlera, L.G.H. Staafa. Impact of designed asymmetries on the effective bandwidth of a backfolded piezoelectric energy harvester.Sensors and Actuators A, 292 (2019) 77-89.

5. Yashwardhan Sahi and Swet Chandan, Finite element analysis of lead-free ceramic based cymbal transducer for energy harvesting, Integrated Ferroelectrics, 212(2020)81-88.

6. Z. Hongduo, Y. Jian, Finite element analysis of cymbal piezoelectric transducers for harvesting energy from asphalt pavement, J. Ceram. Soc. Jpn., 909(2010).118. 